\title{
No change in the prevalence of latent prostate cancer over the last 10 years: a forensic autopsy study in Japan
}

\author{
Masahito Hitosugi ${ }^{1}$, Kenichi Mukaisho ${ }^{2}$, Masahito Kido ${ }^{3}$, Shuichi Kamimura ${ }^{4}$, Satoshi Furukawa ${ }^{1}$, and \\ Hiroyuki Sugihara ${ }^{2}$ \\ ${ }^{1}$ Department of Legal Medicine, Shiga University of Medical Science; ${ }^{2}$ Department of Pathology, Shiga University of Medical Science; \\ ${ }^{3}$ Department of Urology, Jikei University School of Medicine; and ${ }^{4}$ Department of Urology, Utsunomiya Nishigaoka Hospital, Japan \\ (Received 4 August 2017; and accepted 21 August 2017)
}

\begin{abstract}
Although the morbidity rate of prostate cancer has increased with 2.3 times in these 10 years in Japan, little is known about the changes in the prevalence of latent prostate cancer. To understand changes in the prevalence of latent prostate cancer, a retrospective analysis was performed. Forensic autopsy findings from Tochigi Prefecture between September 2012 and February 2014 were collected. Two cross sections, from the base and apex of the prostate, were examined histopathologically. The prevalence of latent prostate cancer was compared with findings from forensic autopsies performed between August 2002 and July 2005 in the same region. The prevalence of latent prostate cancer in both groups was similar, showing an overall prevalence of $13.6 \%$ and $12.2 \%$ and a Gleason score $>6$ of $6.2 \%$ and $7.1 \%$, respectively. When prevalence was compared by cause of death, the values were similar for both groups. The prevalence of latent prostate cancer in this Japanese population did not show any significant change over the past 10 years. The dramatic increase in morbidity rate for prostate cancer could be from the increase in prostate-specific antigen screening and subsequent referral to urologists.
\end{abstract}

Prostate specific antigen (PSA) screening for prostate cancer (PC) is undertaken globally in men aged older than 50 years. Screening has resulted in an increased observance of the incidence of PC and drastically changed the epidemiological characteristics of the disease (12). Because PC is usually a slow progressive disease with a long latent phase, many men with PC die from other causes before they manifest any symptoms of PC (1). In North Ameri$\mathrm{ca}$, although the lifetime risk for a diagnosis of $\mathrm{PC}$ is about $17.0 \%$, the risk for death from $\mathrm{PC}$ is only $3.4 \%$ (5). The detection of PC may therefore have no clinical effect within the lifetime of some pa-

Address correspondence to: Masahito Hitosugi, MD, $\mathrm{PhD}$ Professor, Department of Legal Medicine, Shiga University of Medical Science, Tsukinowa, Seta, Otsu, Shiga 520-2192, Japan

Tel: +81-77-548-2200, Fax: +81-77-548-2201

E-mail: hitosugi@belle.shiga-med.ac.jp tients (6-8).

Determination of the prevalence and characteristics of latent PC may provide an understanding of its natural history. Along with changes in lifestyle, time-related trends must be considered. When considering the prevalence of $\mathrm{PC}$, values are strongly influenced by the specimens available. Because prostate-specific antigen screening or cancer registration does not reflect the true incidence of latent PC, historically, latent PC was mainly assessed by pathological autopsy.

According to recent meta-analyses on autopsy studies, age-adjusted rates of latent PC have remained fairly constant over time $(1,12)$. Recently, some reports suggest that the incidence of PC has increased in Asian countries $(11,14)$. In Japan, according to The Center for Cancer Control and Information Services in Japan, the morbidity rate (per 100000 population) of PC has increased in these ten years, from 47.1 in 2002 to 126.6 in 2011 (4). However, there 
have been little evidences whether the increase of morbidity was owing to the increase of the prevalence of latent $\mathrm{PC}$ or to increased participation in PSA screening and increased referral of patients to the urologists. To clarify the true trend in latent PC in Japan, a forensic autopsy study was performed.

Forensic autopsies are performed to determine causes of unnatural death, including sudden death from disease or external causes, such as accidents, suicide, and homicide. Because such people undertook their normal daily activities immediately before death and had not undergone long-term medical intervention, the natural history of PC can be more accurately evaluated using forensic autopsy specimens.

We previously examined the prevalence of latent PC using forensic autopsy specimens (13). As demographic characteristics play an important role in the incidence of latent $\mathrm{PC}$, we examined changes in the prevalence of latent $\mathrm{PC}$ within the same region of Japan by comparison with recent data.

\section{MATERIALS AND METHODS}

Specimens. Prostate specimens were collected from forensic autopsies performed at Dokkyo Medical University, Tochigi Prefecture, northern Kanto, Japan between September 2012 and February 2014. Bodies severely affected by trauma or decomposition in which there was difficulty removing the entire prostate were excluded. Of the 271 people autopsied over the period, 177 were included in this analysis. General information including age, height, weight, body mass index (BMI), and past medical history was obtained. Each patient's past medical history was reviewed by interviews with their family and referring physicians, if possible. Because forensic autopsies routinely include pathological examinations of the entire body, prostates are usually examined both macroscopically and microscopically. Therefore, collection, processing, and examination of the prostate were not beyond routine forensic autopsy protocol.

At autopsy, the prostate gland was removed and cut into three pieces vertical to the urethra. Two cross sections, one from the base and one from the apex of the prostate, were embedded in paraffin and stained with hematoxylin and eosin using standardized protocols. The study was approved by the Ethics Committee of Shiga University of Medical Science (No. 16-22).

Prostate examination. PC was identified by experienced pathologists (KM, HS). For each specimen, if evaluation was difficult owing to postmortem changes to epithelial cells, it was excluded from further analysis. PC was examined and a Gleason score assigned according to previously established morphologic criteria $(2,3)$. Gleason score is a grading system for PC based on the glandular pattern. Architectural patterns are identified and assigned a grade from 1 (most differentiated) to 5 (undifferentiated). Because the most common and second most common grades were combined in this study, the Gleason scores ranged from $2(1+1)$ to $10(5+5)$. The location of each PC was mapped on a schema of the prostate.

Comparison of the data to previous results. We previously examined the prevalence of latent $\mathrm{PC}$ at forensic autopsy at the same facility between August 2002 and July 2005 (13). Because the main person performing the autopsies (MH) and the methods of examination were the same, we were able to evaluate changes in prevalence over a 10 -year period by comparing the latter results with earlier results. The overall prevalence of latent $\mathrm{PC}$ and the prevalence of PC with a Gleason score $>6$ were compared by manner of death.

Statistical analysis. The chi-squared test was used to compare the prevalence of PC between the two groups. To compare mean age, an F-test was first performed to examine the homogeneity of variance. An unpaired $t$-test was used for equal variance and a Welch's $t$-test was used for unequal variances. A $P$-value $<0.05$ was considered statistically significant.

\section{RESULTS}

\section{Overview}

Among the 177 cases, 15 were excluded because of postmortem changes. For the remaining 162 cases, average postmortem duration was $2.3 \pm 1.8$ days. Age at death ranged from 12 to 94 years, with a mean age of $56.2 \pm 17.8$ years (Fig. 1). Mean BMI was $21.6 \pm 4.9$. Of the 162 cases, $75(46.3 \%)$ died suddenly from disease and $87(53.7 \%)$ from external causes. No cases died of PC. Four patients had been thought to have had PC; however, a biopsy had not been performed, none of these people had a history of prostate surgery, and none had a confirmed diagnosis of PC.

\section{Prevalence of $P C$}

PC was identified in $22(13.6 \%)$ of the 162 autopsied people, who ranged in age between 27 and 88 


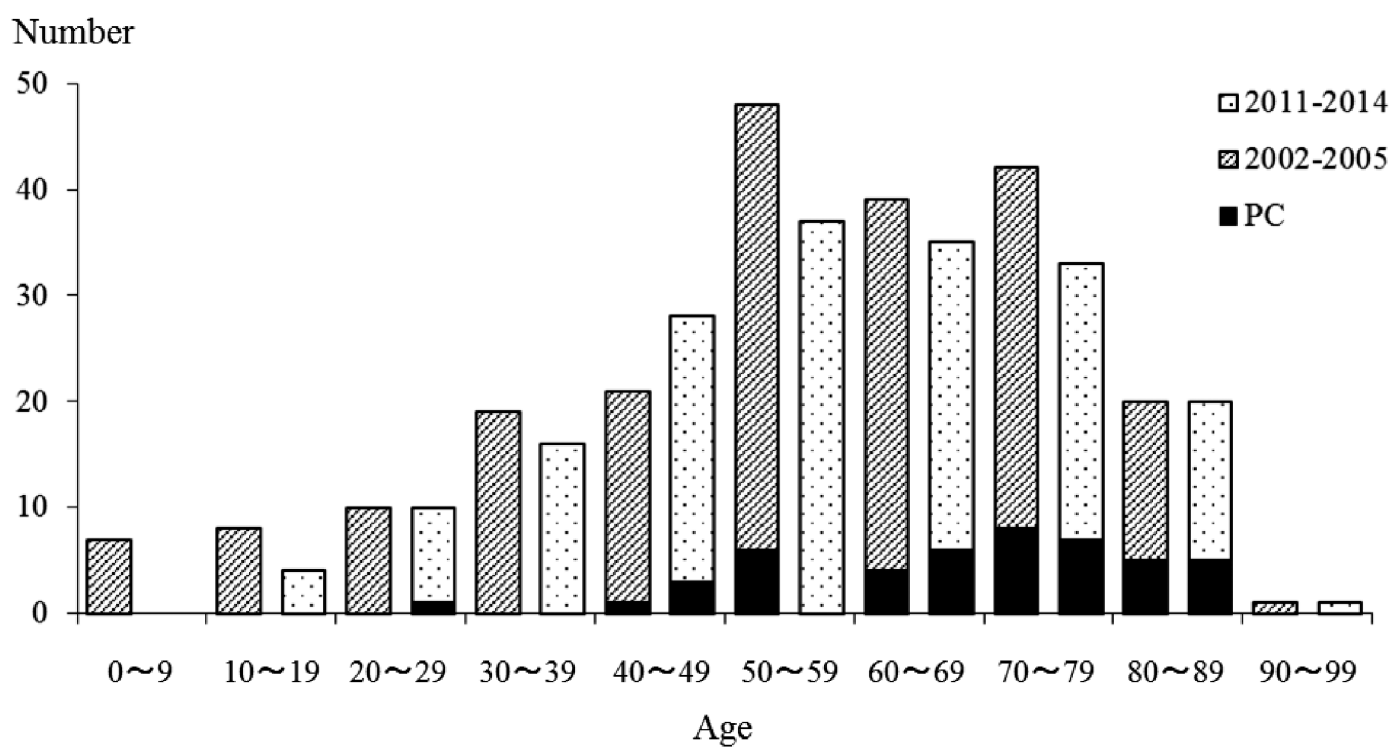

Fig. 1 Comparison of age distribution of all individuals and those with prostate cancer (PC) between two study periods.

A

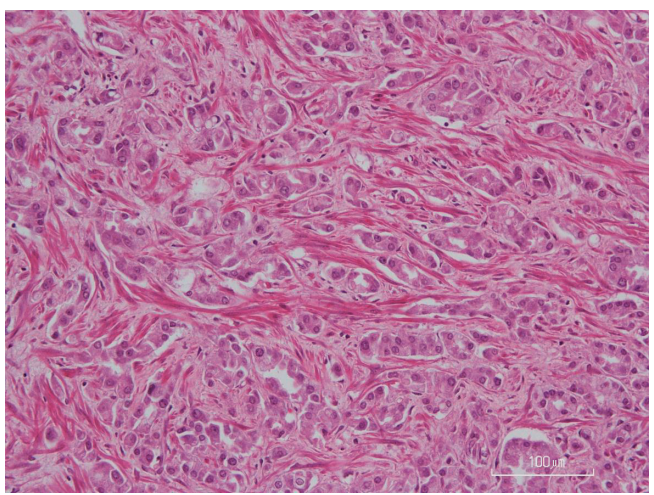

B

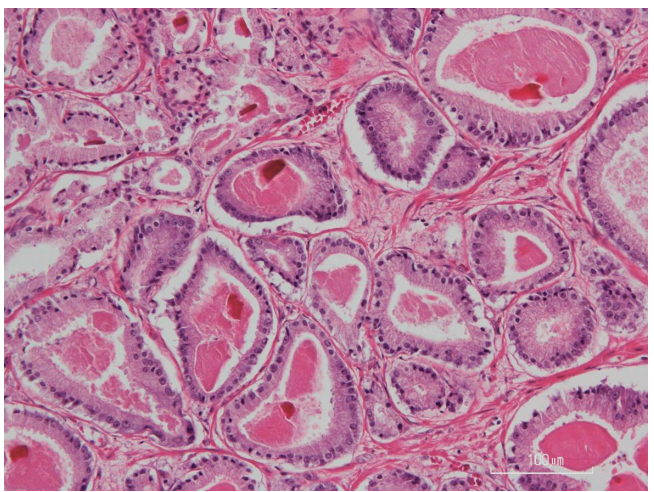

Fig. 2 Representative PC cases with Gleason scores of 9 (A) and 6 (B). Hematoxylin and eosin stain (×200).

years, with a mean age of $67.9 \pm 15.4$ years (Fig. 1). The overall prevalence of PC among people $>49$ years of age was $16.7 \%$. When analyzed by age group, PC was most prevalent among people who had died in their $80 \mathrm{~s}(25.0 \%)$, followed by those in their $70 \mathrm{~s}(21.2 \%), 60 \mathrm{~s}(17.1 \%)$, and $50 \mathrm{~s}(10.9 \%)$ (Fig. 1). The prevalence of PC was similar in people who died from disease or from external causes (13.3\% vs. $13.8 \%$; $P=0.71)$. Representative PC cases including an 83-year-old man with Gleason score 9 and a 76-year-old man with Gleason score 6 are shown in Fig. 2A and 2B, respectively. Both these cases died of thoracic injuries.

Of the 22 PC identified, $10(45.5 \%)$ had a Gleason score $>6$. For these clinically significant PC, prevalence was $6.2 \%$ (Fig. 3). When comparing the prevalence of $\mathrm{PC}$ with a Gleason score $>6$ by cause of death, prevalence was $5.3 \%$ for those who died from disease and $6.9 \%$ for those who died from external causes (Table 1). When the same analysis was restricted to only those aged $>49$ years, prevalence increased to $5.7 \%$ and $9.1 \%$, respectively. Among the four patients thought to have PC, two were diagnosed with PC postmortem, both with Gleason scores of 9 .

\section{Comparison of latest data to previous results}

Both groups (current and previous data) had normal age distributions and similar mean ages (56 and 54 years, respectively, Fig. 1) (13). The prevalence of latent PC by overall age group was similar at $13.6 \%$ for current data and $12.2 \%$ for previous data (statis- 


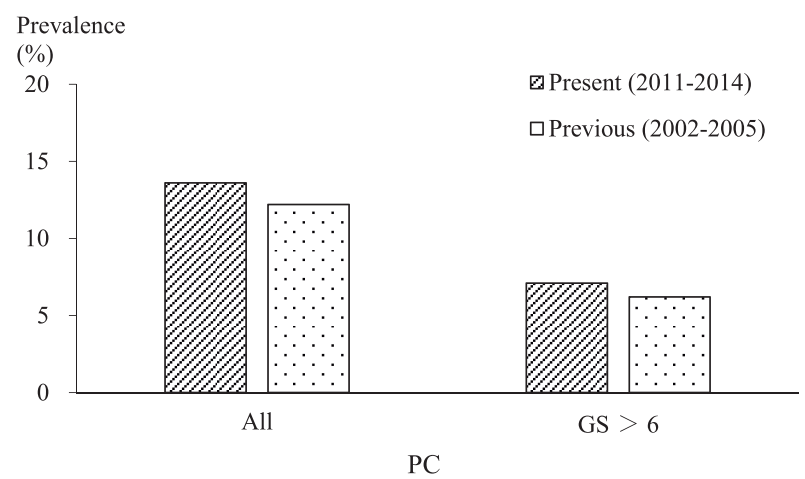

Fig. 3 Comparison of the prevalence of latent prostate cancers, overall and Gleason score (GS) $>6$, between two study periods.

tically not significant, $P=0.71$, Fig. 3). For PC with a Gleason score $>6$, prevalence was also similar at $6.2 \%$ for current data and $7.1 \%$ for previous data (statistically not significant, $P=0.72$, Fig. 3 ). The prevalence of clinically significant latent PC was compared by cause of death and found to be similar for both periods (Table 1).

\section{DISCUSSION}

The present results suggest that the prevalence of latent PC did not change significantly over the 10year study period. From current and previous data, $\mathrm{PC}$ was examined from forensic autopsy cases in the same region, and mean age and age distribution were found to be similar. Therefore, the comparison undertaken should return results with a high accuracy. Although the morbidity of PC has increased with 2.3 times for these ten years in Japan, it is suggested to be because of the increase in prostate-specific antigen screening and the increased referral of patients with high prostate-specific antigen levels to urologists in Japan. As prostate-specific antigen screening is performed in approximately $40 \%$ of men older than 50 years, and as it is likely to increase in the future, it is expected that this discrepancy would become more apparent (17).

Results of this study align with results from meta-analyses of autopsy studies worldwide, which found no strong trends for changes in the prevalence of PC $(2,12)$. However, in Asia, unique observations were found. Yatani et al. compared the prevalence of latent PC from Japanese pathological autopsy cases between the periods 1965-1977 and 1982-1986, and found that the prevalence of PC increased from $22.5 \%$ to $34.6 \%$ between the two periods and that the number of pathologically aggressive tumor phe-
Table 1 Comparison of the prevalence of clinically significant latent prostate cancers (Gleason score > 6) in each cause of death between two study periods

\begin{tabular}{lccc}
\hline & $\begin{array}{c}\text { Present } \\
(2011-2014)\end{array}$ & $\begin{array}{c}\text { Previous } \\
(2002-2005)\end{array}$ & $P$-value \\
\hline Death due to & & & \\
$\quad$ desease & $5.3 \%$ & $5.1 \%$ & 0.96 \\
$\quad$ external causes & $6.9 \%$ & $7.0 \%$ & 0.98 \\
\hline
\end{tabular}

notypes also increased (21). A recent study suggested that the prevalence of latent PC is more frequent in pathological autopsy cases between 2008 and 2013 than in those between 1983 and 1987 (43.3\% and $20.8 \%$, respectively) (14). Diet and metabolic parameters are factors that have been previously discussed as influencing the prevalence of PC $(9,10$, 18). In Japan, the Westernization of dietary habits has progressed remarkably over the last few decades (16). Therefore, as some reports suggest, the recent prevalence may be higher than in the 1960 s to 1980s $(14,20)$. The difference between the previous and present results may be at least partly attributable to differences in the survey periods.

When considering recent trends in nourishment intake in Japan, values are almost constant or only slightly down on those seen between 2002 and 2011; with the average daily intake of carbohydrates going from $297.3 \mathrm{~g}$ to $281.6 \mathrm{~g}$, protein going from $78.6 \mathrm{~g}$ to $73.2 \mathrm{~g}$, lipids going from $58.7 \mathrm{~g}$ to $58.3 \mathrm{~g}$, and sodium chloride going from $12.2 \mathrm{~g}$ to $10.9 \mathrm{~g}$ (16). Therefore, the similar prevalence of latent PC over the 10-year study period is well accordance with the constant or only slightly decreased nutritional trends in Japan over the same time. It is possible that some background characteristics of pathological autopsy subjects may have changed, e.g., mean age, cause of death, and number of patients with cancer, and other studies using pathological autopsies to examine recent 10 -year-trends are required to confirm the present results.

From current data and previous data, mean ages of the autopsied victims were 56 and 54 years, respectively, and overall prevalence of PC was $13.6 \%$ and $12.7 \%$, respectively. In a study in which men had a median age of 63.2 years, PC was detected by biopsy in $24 \%$ (19). A comprehensive summary of 19 published studies found that $\mathrm{PC}$ was diagnosed at autopsy in Asia in $1.8 \%$ for $20-29$ years; $0.9 \%$ for 30-39 years; $2.8 \%$ for $40-49$ years; $7.9 \%$ for $50-59$ years; $14.5 \%$ for $60-69$ years; $21.3 \%$ for 70 79 years; and $28.9 \%$ for $80-89$ years (12). Other autopsy studies have reported that the prevalence of 
PC increases with each decade of age, and this increase is similar for all studies $(1,15)$. These results align with our current and previous data.

Clinically significant PC has been defined as a tumor with a Gleason score $>6$. PC that does not meet this criterion are regarded as clinically insignificant and unlikely to adversely affect the health of the patient. In the current study, the prevalence of PC with a Gleason score $>6$ was $6.2 \%$, which was similar to data from 10 years ago. This value increased to $7.5 \%$ if patients were aged 50 years or older. PC with a Gleason score $>6$ was $43.5 \%$ in all latent PC, which is in accordance with a recent pathological autopsy study that found that 24 of $55(43.5 \%)$ latent PC patients had a Gleason score of 7 or higher (14).

Some points need to be kept in mind when comparing the prevalence of PC between our study and other studies. For example, the studies included in the meta-analyses performed full slicing of the prostate gland into thin sections $(1,12,15)$. In the current study, the prostate was only cross sectioned into two pieces, which may result in our data underestimating the prevalence. Also, most studies in the meta-analyses did not include clinically diagnosed PC cases, and may mean that the data underestimate the true cumulative incidence of total PC (1). At the time of the pathological autopsy, many had been on medical interventions such as carcinostatics, were elderly at the time of death, or had cancers other than PC, all factors which might increase the prevalence of PC. We found that the prevalence of PC was similar in both men who died suddenly from disease and from external causes, indicating that the use of forensic autopsy specimens, including those of people who died suddenly from disease or from external causes, can be used to determine the prevalence of latent PC. It is suggested that, when comparing the prevalence of latent $\mathrm{PC}$, using forensic autopsy data is suitable.

In this study, the postmortem duration was 2.3 days, longer than other pathological autopsy studies. Most forensic autopsies were performed on people who were found dead and, in some cases, people were found in places with a low temperature. Despite the long postmortem duration, we were able to evaluate microscopic changes of the prostate for some of those people who were found in cold temperatures. Cases were not chosen based on any limitations on postmortem duration. Pathologists determined, in a blind manner, whether microscopic observation was possible. Therefore, it is suggested that our method did not bias the results. It is considered that the most crucial possible limitation of the current study was the sectioning of each prostate specimen into cross sections. Section thickness during prostate processing may have influenced the prevalence of PC. Furthermore, other pathologic information, i.e., tumor volume and location, is lacking. However, as the objective of the study was to compare the prevalence of latent PC, and as this method was used for both current and previous comparison data, it is suggested that this method does not influence the results.

The prevalence of latent PC did not change significantly over the 10-year study period in this population of Japanese people. The dramatic increase in morbidity rate for PC is considered to be because of the increase in prostate-specific antigen screening and subsequent referral to urologists. As forensic autopsy specimens are suitable to determine latent $\mathrm{PC}$, trends and racial and regional differences in latent PC should be examined in the future.

\section{Acknowledgements}

The authors gratefully acknowledge Akari Uno, the clinical technicians who provided the pathological specimens.

\section{CONFLICTS OF INTEREST}

The authors declare that they have no conflicts of interest.

\section{REFERENCES}

1. Bell KJ, Del Mar C, Wright G, Dickinson J and Glasziou P (2015) Prevalence of incidental prostate cancer: A systematic review of autopsy studies. Int J Cancer 137, 1749-1757.

2. Bostwick, DG, Amin MB, Dundore P, Marsh W and Schltz DS (1993) Architectural patterns of high-grade prostatic intraepithelial neoplasia. Hum Pathol 24, 298-310.

3. Bostwick DG, Graham SD Jr, Napalkov P, Abrahamsson PA, di Sant'agnese PA, Algaba F, Hoisaeter PA, Lee F, Littrup P, Mostofi FK, Abrahamsson PA, Denis L, Di Sant'agnese PA, Schroeder F, Algaba F, Murphy GP and Hoisaeter PA (1993) Staging of early prostate cancer: a proposed tumor volumebased prognostic index. Urology 41, 403-411.

4. CANCER STATISTICS IN JAPAN, Center for Cancer Control and Information Services, National Cancer Center. http:// ganjoho.jp/reg_stat/statistics/index.html

5. Center MM, Jemal A, Lortet-Tieulent J, Ward E, Ferlay J, Brawley $\mathrm{O}$ and Bray $\mathrm{F}$ (2012) International variation in prostate cancer incidence and mortality rates. Eur Urol 61, 1079 1092.

6. Ciatto S, Gervasi G, Bonardi R, Frullini P, Zendron P, Lombardi C, Crocetti E and Zappa M (2005) Determining overdiagnosis by screening with DRE/TRUS or PSA (Florence pilot studies, 1991-1994). Eur J Cancer 41: 411-415.

7. Draisma G, Boer R, Otto SJ, van der Cruijsen IW, Damhuis RA, Schröder FH and de Koning HJ (2003) Lead times and overdetection due to prostate-specific antigen screening: esti- 
mates from the European randomized study of screening for prostate cancer. $J$ Natl Cancer Inst 95, 868-878.

8. Etzioni R, Penson DF, Legler JM, di Tommaso D, Boer R, Gann PH and Feuer EJ (2002) Overdiagnosis due to prostate-specific antigen screening: lessons from U.S. prostate cancer incidence trends. J Natl Cancer Inst 94, 981-990.

9. Hayashi N, Matsushima M, Kido M, Naruoka T, Furuta A, Furuta N, Takahashi H and Egawa S (2014) BMI is associated with larger index tumors and worse outcome after radical prostatectomy. Prostate Cancer Prostatic Dis 17, 233-237.

10. Hayashi N, Matsushima M, Yamamoto T, Sasaki H, Takahashi $\mathrm{H}$ and Egawa S (2012) The impact of hypertriglyceridemia on prostate cancer development in patients aged $\geq 60$ years. BJU Int 109, 515-519.

11. Ito K (2014) Prostate cancer in Asian men. Nat Rev Urol 11, 197-212.

12. Jahn JL, Giovannucci EL and Stampfer MJ (2015) The high prevalence of undiagnosed prostate cancer at autopsy: implications for epidemiology and treatment of prostate cancer in the Prostate-specific Antigen-era. Int J Cancer 137, 27952802.

13. Kido M, Hitosugi M, Ishii K, Kamimura S and Joh K (2015) Latent prostate cancer in Japanese men who die unnatural deaths: A forensic autopsy study. Prostate 75, 917-922.

14. Kimura T, Takahashi H, Okayasu M, Kido M, Inaba H, Kuruma H, Yamamoto T, Furusato B, Furusato M, Wada T and Egawa S (2016) Time trends in histological features of latent prostate cancer in Japan. J Urol 195, 1415-1420.
15. Leal J, Hamdy F and Wolstenholme J (2014) Estimating age and ethnic variation in the histological prevalence of prostate cancer to inform the impact of screening policies. Int $J$ Urol 21, 786-792.

16. National Health and Nutrition Survey, Ministry of Health, Labour and Welfare. http://www.mhlw.go.jp/bunya/kenkou/eiyou/ h26-houkoku.html

17. Sawada K, Kitagawa Y, Ito K, Takeda Y, Mizokami A and Namiki M (2014) Cumulative risk of developing prostate cancer in men with low $(\leq 2.0 \mathrm{ng} / \mathrm{mL})$ prostate-specific antigen levels: a population-based screening cohort study in Japan. Int J Urol 21, 560-565.

18. Tominaga $\mathrm{S}$ and Kuroishi $\mathrm{T}$ (1997) An ecological study on diet/nutrition and cancer in Japan. Int J Cancer 10, 2-6.

19. Thompson IM, Goodman PJ, Tangen CM, Lucia MS, Miller GJ, Ford LG, Lieber MM, Cespedes RD, Atkins JN, Lippman SM, Carlin SM, Ryan A, Szczepanek CM, Crowley JJ and Coltman CA Jr (2003) The influence of finasteride on the development of prostate cancer. $N$ Engl J Med 349, 215-224.

20. Watanabe M, Nakatama T, Shiraishi T, Stemmermann GN and Yatani R (2000) Comparative studies of prostate cancer in Japan versus the United States. A review. Urol Oncol 5, 274-283.

21. Yatani R, Shiraishi T, Nakakuki K, Kusano I, Takanari H, Hayashi T and Stemmermann GN (1988) Trends in frequency of latent prostate carcinoma in Japan from 1965-1979 to 1982-1986. J Natl Cancer Inst 80, 683-687. 